\title{
POSITION STATEMENT: VRE screening and contact precautions
}

This position statement was developed by the Standards and Guidelines Committee.

Chair: Madeleine Ashcroft

Principal Authors: Standards and Guidelines Committee

\section{Publication Date}

Original: 2012 November

Revised: 2019 November

\section{BACKGROUND}

Vancomycin resistant enterococci (VRE) are present in many healthcare facilities across Canada to varying degrees, usually as rectal colonization [1]. VRE bacteraemia is associated with greater hospital mortality and length of stay than vancomycin-sensitive enterococcal (VSE) bacteraemia [2]. In recent years, some Canadian healthcare facilities have decided to reduce or stop screening as well as the use of contact precautions as a VRE control strategy. Others continue to support current guideline recommendations for VRE surveillance and the use of additional precautions [3].

\section{POSITION STATEMENT}

IPAC Canada recognizes that while there are various bodies of expert opinion on VRE control, recent Ontario studies [3-7] support ongoing screening and contact precautions. Decisions regarding screening and contact precautions should be based on local epidemiology, and guided by regional and provincial recommendations and requirements [4-9]. Further, any changes to practice should be implemented to improve patient care and not be used as a cost-cutting measure. These changes should only be considered in the context of an infection prevention and control program already meeting or exceeding best practices (including hand hygiene, environmental cleaning, routine practices and additional precautions).

For those healthcare facilities that are considering or have implemented a reduction in VRE control strategies, IPAC Canada recommends an approach that considers the following:

- Epidemiologic investigation and risk assessment for VRE infections;

- Consultation with staff and client groups, including high-risk wards/clinics;

- Consultation with institutional stakeholders;

- Discussion with other internal and external stakeholders, including the health region; and
- An enhanced communication strategy addressing multiple contingencies (e.g., continued surveillance may show a need to return to previous practices)

Further, IPAC Canada recommends that any savings incurred from decreased screening and contact precautions is reinvested in the following activities (as determined by the risk assessment above):

- Education on Routine Practices

- Environmental cleaning;

- Hand hygiene;

- Antimicrobial stewardship;

- Monitoring of healthcare-acquired infections (HAls); and

- Other activities deemed important for infection control and prevention.

Decreased surveillance of VRE results in a paucity of information regarding colonization. Any reduction in screening and contact precautions should be accompanied by close monitoring of all VRE culture-positive HAls to ensure that undue harm is not incurred as a result. In the event that harm is found, institutions should be prepared to return to previous policies. It is also highly recommended that those institutions that choose to change their strategy communicate their experiences to other members of the infection control community for future policy making.

\section{STAKEHOLDERS}

Infection Prevention and Control Professionals, healthcare workers, and their clients (the Canadian public).

\section{REFERENCES}

1. Ofner-Agostini $M$, Johnston BL, Simor AE, Embil J, Matlow A, Mulvey M, et al. (2008). Vancomycin-Resistant Enterococci in Canada: Results from the Canadian Nosocomial Infection Surveillance Program, 1999-2005. Infect Control Hosp Epidemiol, 29(3), 271-4. doi: 10.1086/528812 
2. Prematunge C, MacDougall C, Johnstone J, Adomako K, Lam F, Robertson J, et al. (2016). VRE and VSE bacteremia outcomes in the era of effective VRE therapy: A systematic review and meta-analysis. Infect Control Hosp Epidemiol, 37(1),26-35. doi:10.1017/ice.2015.228

3. Buick S, Joffe AM, Taylor G, Conly J. (2015). A consensus development conference model for establishing health policy for surveillance and screening of antimicrobialresistant organisms. Clin Infect Dis, 60(7),1095-101. Doi:10.1093/cid/ciu1168

4. Johnstone J, Policarpio ME, Lam F, Adomako K, Prematunge C, Nadolny E, et al. (2017). Rates of blood cultures positive for vancomycin-resistant Enterococcus in Ontario: a quasiexperimental study. CMAJ Open, 5(2),e273-80. doi:10.9778/ cmajo.20160121

5. A PHO/IPAC memorandum (Update on rising VRE bacteremia rates in Ontario). 23 June 2017. Retrieved from: http://www.publichealthontario.ca/en/eRepository/VRE_ Letter_Research_Update.pdf (accessed 31 Oct 2019).
6. Ontario Agency for Health Protection and Promotion (Public Health Ontario), Provincial Infectious Diseases Advisory Committee. Evidence review and revised recommendations for the control of vancomycin-resistant enterococci in all Ontario health care facilities. Toronto, ON: Queen's Printer for Ontario; 2019. Retrieved from: https://www.publichealthontario.ca/-/media/documents/ recommendations-vre.pdf?la $=$ en (accessed 31 Oct 2019).

7. Johnstone J, Chen C, Shing E, Adomako K, Garber G, Sander B. The Economic Burden of Vancomycin-Resistant Enterococcus (VRE) Bacteremia: A Population-Based Matched Cohort Study. Poster presented at: 41st Annual North American Meeting of the Society for Medical Decision Making. 2019 Oct 20-23; Portland, OR.

8. Institut national de santé publique du Québec (INSPQ). Healthcare-associated infections provincial surveillance program: Highlights, discussions and orientations 20142015. September 2015. Retrieved from https://www. inspq.qc.ca/pdf/publications/2172_highlights_discussions orientations.pdf (accessed 31 Oct 2019).

9. Han G. The impact of vancomycin-resistant Enterococci (VRE): Policy and practice changes in BC. Retrieved from https:/www.picnet.ca/wp-content/uploads/Impact-of-VREchanges-in-BC.pdf (accessed 31 Oct 2019). 